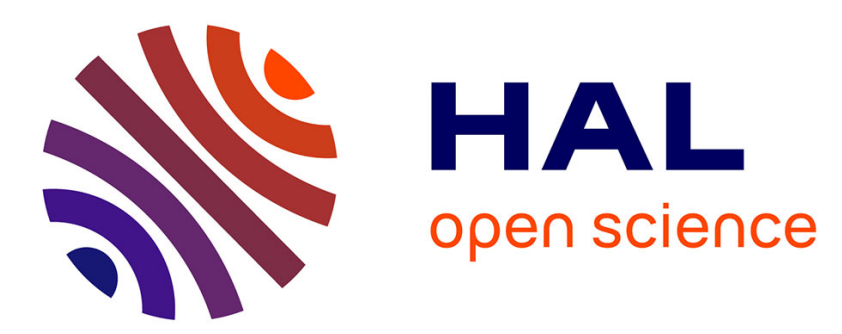

\title{
Statistical approach for a hyper-visco-plastic model for filled rubber: experimental characterization and numerical modeling
}

Jean-Marc Martinez, Adnane Boukamel, Stéphane Méo, Stéphane Lejeunes

\section{- To cite this version:}

Jean-Marc Martinez, Adnane Boukamel, Stéphane Méo, Stéphane Lejeunes. Statistical approach for a hyper-visco-plastic model for filled rubber: experimental characterization and numerical modeling. European Journal of Mechanics - A/Solids, 2011, 30 (6), pp.1028-1039. 10.1016/j.euromechsol.2011.06.013 . hal-00614912

\author{
HAL Id: hal-00614912 \\ https://hal.science/hal-00614912
}

Submitted on 18 Apr 2018

HAL is a multi-disciplinary open access archive for the deposit and dissemination of scientific research documents, whether they are published or not. The documents may come from teaching and research institutions in France or abroad, or from public or private research centers.
L'archive ouverte pluridisciplinaire HAL, est destinée au dépôt et à la diffusion de documents scientifiques de niveau recherche, publiés ou non, émanant des établissements d'enseignement et de recherche français ou étrangers, des laboratoires publics ou privés. 


\title{
Statistical approach for a hyper-visco-plastic model for filled rubber: experimental characterization and numerical modeling
}

\author{
J.M. Martinez ${ }^{\mathrm{a}}$, A. Boukamel ${ }^{\mathrm{a}, *}$, S. Méo ${ }^{\mathrm{b}}$, S. Lejeunes ${ }^{\mathrm{a}}$ \\ ${ }^{a}$ Laboratoire de Mécanique et d'Acoustique, CNRS UPR-7051, 31 chemin Joseph-Aiguier, 13402 Cedex 20, Marseille, France \\ ${ }^{b}$ Laboratoire de Mécanique et Rhéologie, EPU Dept Productique, BP 407, F37204 Cedex 3, Tours, France
}

\begin{abstract}
This paper presents a campaign of experimental tests performed on a silicone elastomer filled with silica particles. These tests were conducted under controlled temperatures (ranging from $-55^{\circ} \mathrm{C}$ to $+70^{\circ} \mathrm{C}$ ) and under uniaxial tension and in shearing modes. In these two classes of tests, the specimens were subjected to cyclic loading at various deformation rates and amplitudes and relaxation tests at various levels of deformation. A statistical hyper-visco-elasto-plastic model is then presented, which covers a wide loading frequency spectrum and requires indentifying only a few characteristic parameters. The method used to identify these parameters consists in performing several successive partial identifications with a view to reducing the coupling effects between the parameters. Lastly, comparisons between modeling predictions and the experimental data recorded under harmonic loading, confirm the accuracy of the model in a relatively wide frequency range and a large range of deformations.
\end{abstract}

Key words: Hyperviscoplastic behavior, Rheological model, Payne effect, Gent-Fletcher effect, Filled elastomer.

\section{Introduction}

Elastomers belong to the high polymer family i.e. they consist of macromolecular chains of various lengths, with and without ramifications. This structure confers on these materials a low level of rigidity and a high level of deformability. In addition, the reinforcement of these materials with fillers accentuates their dissipative behavior. Because of these properties, especially their damping capacity, these materials are widely used in industry. The application on which this study focuses is that of the drag dampers for helicopters. These parts connect helicopter blades to the rotor and attenuate the drag movement. Designing these parts, which are often related to safety, imposes a guarantee of high reliability under extreme operating conditions (dynamic loading with multi-frequency and large amplitudes, thermal constraints, etc). Meeting

\footnotetext{
* Correcponding author

Email addresses: adnane.boukamel@ec-marseille.fr (A. Boukamel)

URL: http://www.Ima.cnrs-mrs.fr (A. Boukamel)
}

Preprint submitted to European Journal of Mechanics A/Solids 
these specifications requires good knowledge of the mechanical behavior of the constitutive materials. In addition, the behavior of an elastomer can depend heavily on the temperature, the degree of cross-linking and the type of particles incorporated (carbon or silica), etc.

During recent decades, several approaches have been used by previous authors to model various behavioral aspects of elastomers:

- To describe the static behavior of the material, a hyperelastic approach was used in: Treloar (1943, 1957) where statistical models were proposed; and Mooney (1940); Rivlin (1958); Hart-Smith (1966); Ogden (1972), which involved the use of phenomenological approaches.

- Some authors have used the damage mechanics approach to describe the softening behavior occurring during the first loading cycles, which is known as the Mullins effect, see Mullins (1947); Harwood et al. (1967). A theoretical framework was proposed by Govindjee and Simo $(1991,1992)$ in the case of a hyperelastic behavior. A similar approach was described in Simo (1987); Miehe (1995) in the case of a viscoelastic material.

- In Holzapfel and Reiter (1995); Holzapfel and Simo (1996a,b); Lion (1997), a thermomechanical coupling model was developed, which takes into account the temperature dependence of the mechanical characteristics and describes the temperature changes resulting from the mechanical dissipation.

- Furthermore, to model the viscoelastic effects of these materials, a framework of the Finite Non Linear Viscoelasticity has been proposed by several authors. These models can be classified in the following groups, depending on the type of formulation used:

Those using an integral approach, which were mainly developed for modeling non linear materials with evanescent memory. These approaches describe the behavior of the material using equations giving the stress tensor in terms of the strain history. Rivlin (1958); Coleman (1964); Christensen (1971); Coleman and Noll (1961); Lianis (1963); Chang et al. (1978); Morman (1988).

Those using a differential approach, based on the concept of intermediate states commonly used to describe finite elastic-plastic deformations (see Sidoroff $(1973,1974)$ ). Defining intermediate states provides the internal variables needed to describe the behavior. This approach can be said to be an extension of rheological models in the case of large strains: Sidoroff (1977); Le Tallec (1990); Le Tallec and Rahier (1994); Leonov (1992). The local state method, Lemaitre and Chaboche (1996), provides the theoretical framework of this formulation, and the internal variables are provided by the intermediate states.

And those using micro-physically motivated models for filled elastomers, which are often based on hypotheses about the interactions between the agglomerates of fillers and the gum matrix: Drozdov (2001a,b); Drozdov and Dorfmann (2002, 2003); Drozdov et al. (2004), or about the mechanisms 
underlying the deformation and rearrangement of the macro-molecular network: Tanaka and Edwards (1992); Drozdov (1998, 2000); Reese (2003).

In this paper, a meso-physically motivated approach is used to model the response of the material, in large strain and at various frequencies and temperatures. A statistical approach is then proposed to develop a model based on the generalization of an assembly of rheological models. The advantage of this statistical rheological model is that it can be used to simulate the behavior of the material in a wide frequency range while requiring only a few parameters to be identified.

First we present the results of a series of experimental tests, which were carried out on a silicone elastomer filled with silica. These tests were uniaxial tension and shear tests and were performed under controlled temperature (ranging from $-55^{\circ} \mathrm{C}$ to $+70^{\circ} \mathrm{C}$ ) and under various loading conditions (Relaxation tests, quasistatic and dynamic loading at various strain rates). The results show the dependence of the behavior of the material on the temperature, as well as on the strain-rate (Fletcher-Gent effect, see Fletcher and Gent (1953)) and the amplitude of the strain (Payne effect, see Harwood et al. (1967)). The constitutive model is then developed on the basis of the fundamental principle of thermodynamics of continuous media, adapted to finite strain theory. Using the concept of intermediate configurations (multiplicative decomposition of the deformation gradient) and in line with the theory of thermodynamics of irreversible processes, and under the hypothesis of the normal dissipation depending only on the internal variables, the constitutive equation and the flow rules are obtained. A statistical approach is then applied, in order to extend this rheological model to a wide range of strain rates and to account for the plastic behavior of the material. In the following section, this statistical hyper-visco-plastic model is analyzed in the case of simple loads, with a view to propose a strategy for identifying its parameters. For this purpose, analytical solutions are developed to simulate the relaxation response and the hardening test, respectively. In the case of cyclic loading, a semianalytical response was obtained using a symbolic and numeric computation software. These identifications were performed at various temperatures. Lastly, using the semi-analytical solution under sinusoidal shear loading conditions at various frequencies and amplitudes, the effect of parameters such as the temperature, frequency and loading amplitude on the harmonic response of the elastomer are analyzed.

\section{Experimental analysis}

\subsection{Description of the experimental tests}

An experimental campaign was conducted on a silicone (dimethyl-vinyl-siloxan vulcanized by peroxide) reinforced with silica particles. The glass transition temperature of this elastomer is approximately $-105^{\circ} \mathrm{C}$. The following tests were carried out:

- Uniaxial tensile tests on specimens with a dumbbell shape (H2 according to standard NF T46-002), to determine the quasi-static behavior and the relaxation response of the material. 


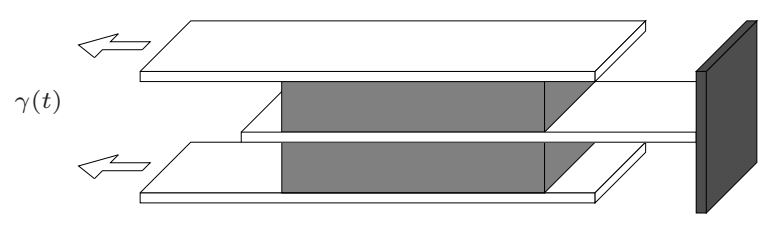

Figure 1: Double-shearing specimen.

- Shear tests on Double-Shearing specimens (DS, see Figure 1). These specimens were successively subjected to: a quasi-static loading-unloading cycle; relaxation tests at various shearing levels; triangular cyclic loading, at various strain rates (from $0.03 s^{-1}$ to $10 s^{-1}$ ) and various amplitudes $(12.5 \%, 25 \%$ and $50 \%$ ).

All these tests were performed under controlled temperatures (ranging from $-55^{\circ} \mathrm{C}$ to $+70^{\circ} \mathrm{C}$ ) in a climatic chamber cooled by injecting nitrogen and heated with an electrical resistance and the airflow. In the tensile tests, monitoring and deformation measurements were performed with a laser extensometer.

Remark 1 (Mullins effect) To eliminate the Mullins effect (see Mullins (1947); Harwood et al. (1967)) and therefore to characterize the behavior of the stabilized material, a softening process was first induced by applying about ten cycles with an amplitude greater than the maximum strain imposed during the series of tests.

Remark 2 (Temperature stabilization) To avoid errors in the temperature measurement, a waiting period of ten minutes was fixed between each characterization test to allow the temperature to reach equilibrium inside the specimen. The characterization time was sufficiently short to avoid a too strong self-heating phenomena in the specimen.

\subsection{Experimental results}

Relaxation tests: In the relaxation tests, the specimen was subjected to various strain levels: $25 \%, 50 \%$ and 100\% under tension loading; 20\%, 30\% and 54\% under shear strain. The response of the material is described by the evolution of the normalized stress ${ }^{1}$ versus time. The curves presented in Figure 2(a) and Figure 2(c) show that at temperatures above the ambient temperature, the evolution of these stresses during relaxation was always independent of the strain amplitude. At these temperatures, the relaxation mechanism seems independent of the strain level, under both tension and shear loading; whereas at lower temperatures, the responses doesn't show the same linearity of the stress depending on the deformation, especially in the case of uniaxial tension tests (see Figure 2(b)). The graphs in Figure 2(b) and Figure 2(d)

\footnotetext{
${ }^{1}$ For relaxation tests, normalized stress is obtained by dividing the total stress by the instantaneous stress.
} 


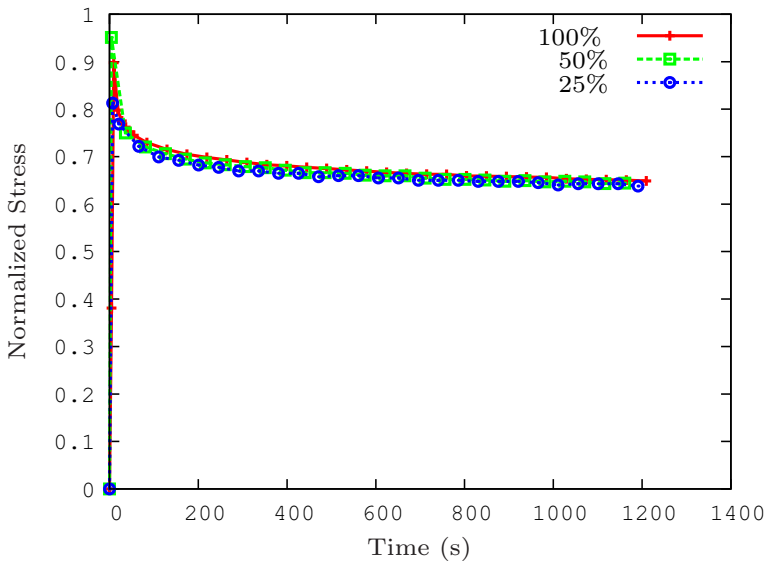

(a) Effects of the elongation under tension loading $(T=$ $\left.25^{\circ} \mathrm{C}\right)$

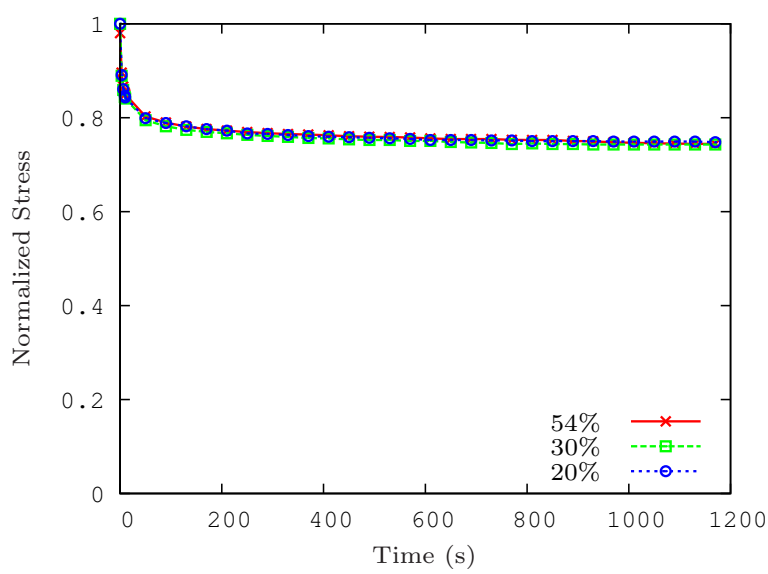

(c) Effects of the strain amplitude under shear loading $\left(T=25^{\circ} \mathrm{C}\right)$.

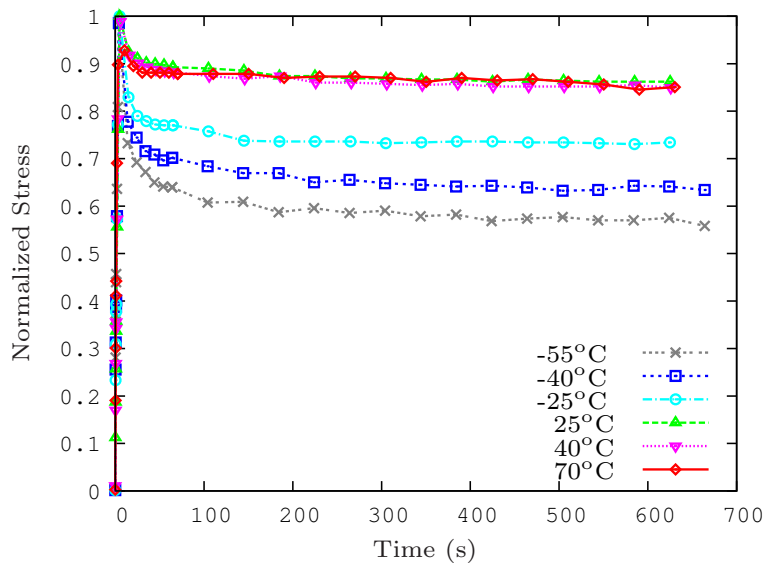

(b) Effects of the temperature under tension loading.

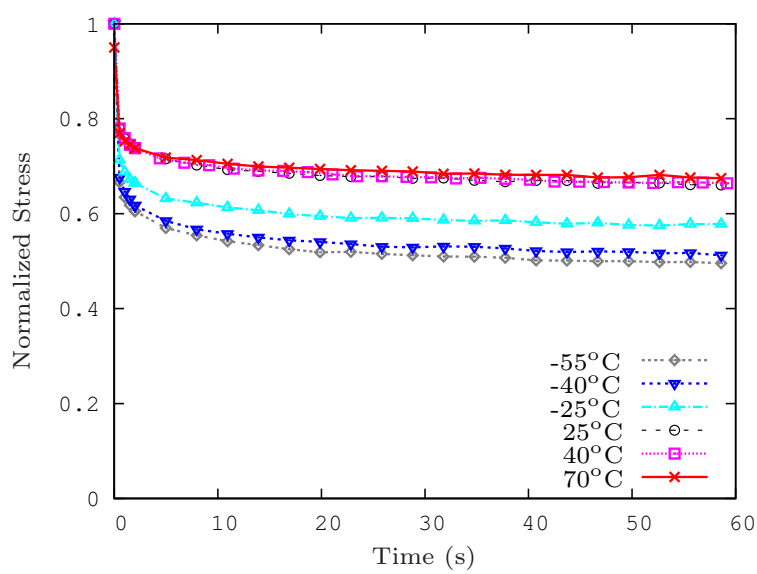

(d) Effects of the temperature under shear loading.

Figure 2: Response of the material in relaxation tests: first Piola-Kirchoff stress $v s$ time. The vertical axis corresponds to the total stress divided by the instantaneous stress (normalized stress).

show the dependence of the relaxation response on the temperature. The relaxation response was therefore more sensitive to the temperature in the $\left[-55^{\circ} \mathrm{C},-25^{\circ} \mathrm{C}\right]$ range than at higher temperatures (above $25^{\circ} \mathrm{C}$ ).

Quasi-static shear response: The quasi-static test was a loading-unloading test, performed at low strain rate $\left(\dot{\gamma}=0.03 s^{-1}\right)$ and for three shear amplitudes $\left(\gamma_{\max }=12.5 \%, 25 \%\right.$ and $\left.50 \%\right)$. The stress-strain curves given in Figure 3 show that even at low rates of deformation, the material shows dissipative behavior. It will therefore be necessary to take the plasticity into account when developing the constitutive model. 


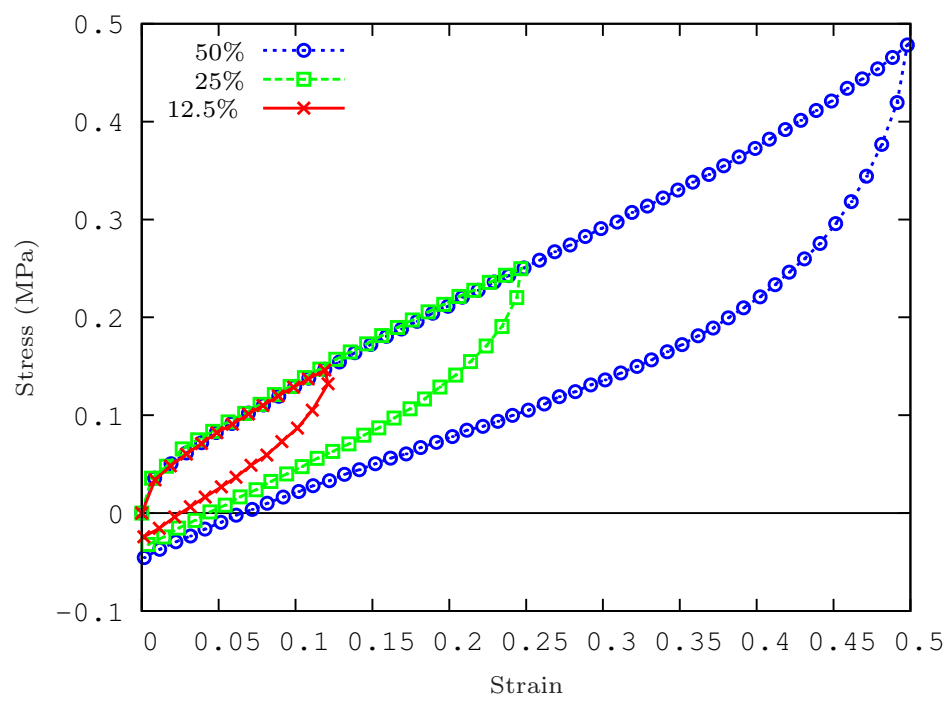

Figure 3: Quasi-static responses recorded in loading-unloading shear tests: first Piola-Kirchoff stress vs shear strain (shear rate: $\dot{\gamma}=0.03 s^{-1}$, temperature: $\left.T=25^{\circ} \mathrm{C}\right)$.

Cyclic tests: Cyclic tests were carried out under triangular loading conditions with gradually increasing amplitude. At each amplitude, a dozen cycles were applied. The values of the parameters in this set of tests were as follows:

- Temperature $(T): 70^{\circ} \mathrm{C}, 40^{\circ} \mathrm{C}, 25^{\circ} \mathrm{C},-25^{\circ} \mathrm{C},-40^{\circ} \mathrm{C}$ and $-55^{\circ} \mathrm{C}$.

- Shear rate $(\dot{\gamma}): 0.03 s^{-1}, 0.1 s^{-1}, 0.3 s^{-1}, 1 s^{-1}, 3 s^{-1}$ and $10 s^{-1}$.

- Shear amplitude $\left(\gamma_{\max }\right): 12.5 \%, 25 \%$ and $50 \%$.

Figures 4(a) and 5 show the effects of the loading amplitude on the stabilized response. Qualitatively, these responses show the strong non-linearity at high amplitudes. In addition, it is worth noting the decrease in the global stiffness observed when the strain amplitude increases. These results therefore clearly confirm that this phenomenon, which is known as the Payne effect, is more pronounced at low temperatures (see Figure 5(a)). In previous studies, this softening has often been attributed to the plastic behavior of elastomers reinforced with fillers.

As with other visco-elastic polymer materials, the influence of the strain rate was more classical (see Figures 4(b) and 6) at all the temperatures tested: an increase in the global stiffness and the cyclic dissipation with the strain rate were clearly observed.

Lastly, the hysteresis loops presented in Figure 7 show the strong influence of the temperature on the behavior of the material. It can be seen in particular that: a softening and a decrease in the cyclic dissipation occurs as the temperature increases; at low temperatures the hysteresis loop shows a nonlinear behavior, which is characterized by: the angular point, the stiffening observed at the end of the cycle and the contraction 


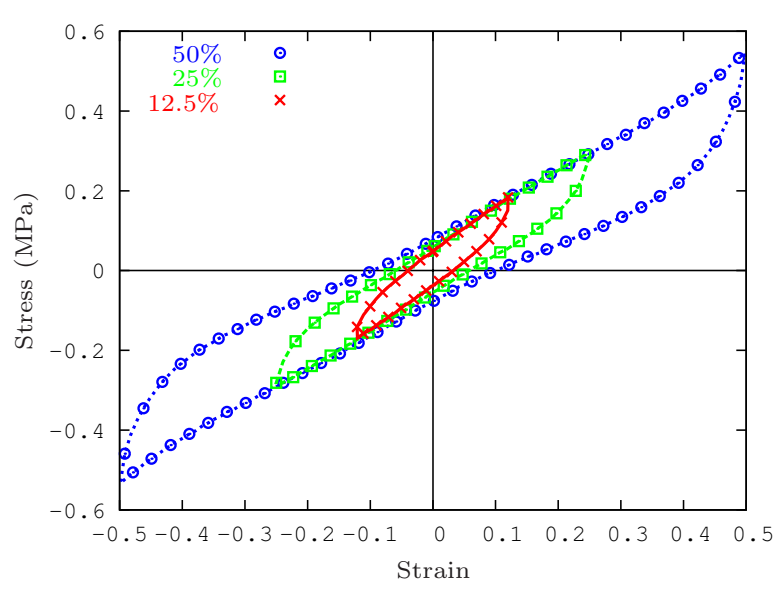

(a) Stabilized cycles $\left(T=25^{\circ} \mathrm{C}, \dot{\gamma}=0.3 \mathrm{~s}^{-1}\right)$, effect of the strain amplitude.

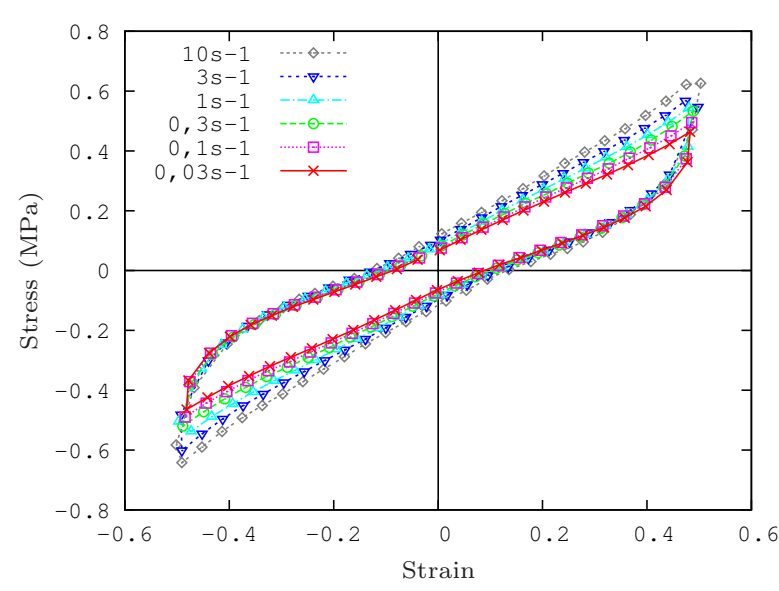

(b) Stabilized cycles $\left(T=25^{\circ} \mathrm{C}, \gamma_{\max }=50 \%\right)$, effect of the strain rate.

Figure 4: Cyclic responses in triangular shear tests: first Piola-Kirchoff stress vs shear strain (temperature: $T=25^{\circ} \mathrm{C}$ ). The first cycles has been removed to keep only stabilized responses.

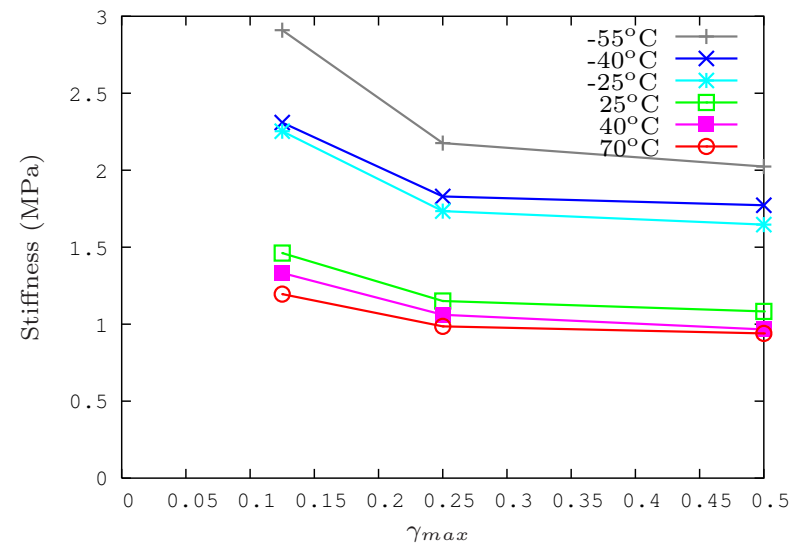

(a) Global stiffness vs strain amplitude

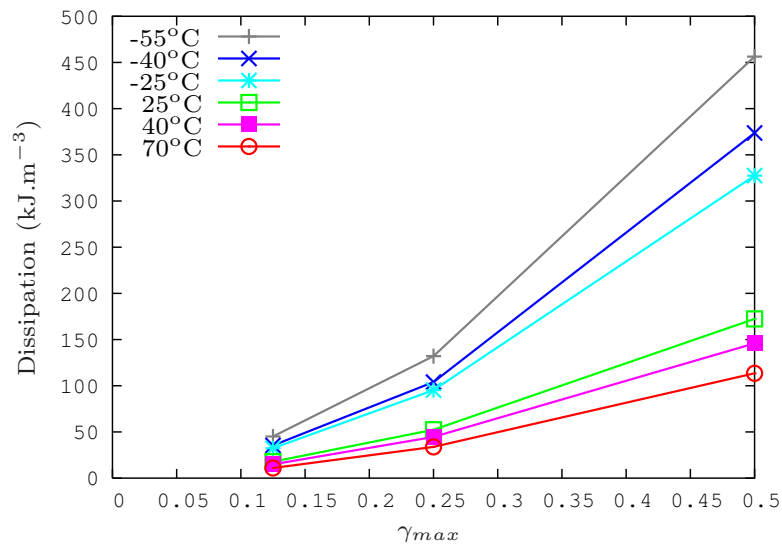

(b) Cyclic dissipation vs strain amplitude

Figure 5: Effects of the strain amplitude on the response of the material to triangular cyclic shearing tests for various temperatures $\left(\dot{\gamma}= \pm 0.3 \mathrm{~s}^{-1}\right)$.

of the loop at zero strain. These non-linear features, which are more pronounced at low temperatures, are consistent with plastic behavior. These results therefore confirm that plastic behavior begins to predominate when the material approaches the glass transition point.

In conclusion, the following aspects of the behavior have to be taken into account in the model:

- The geometric non-linearities due to large strains. 


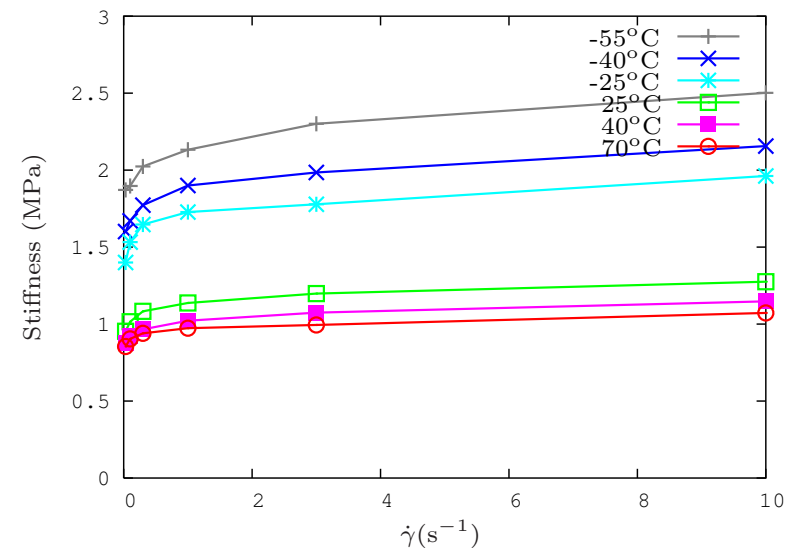

(a) Global stiffness vs strain rate

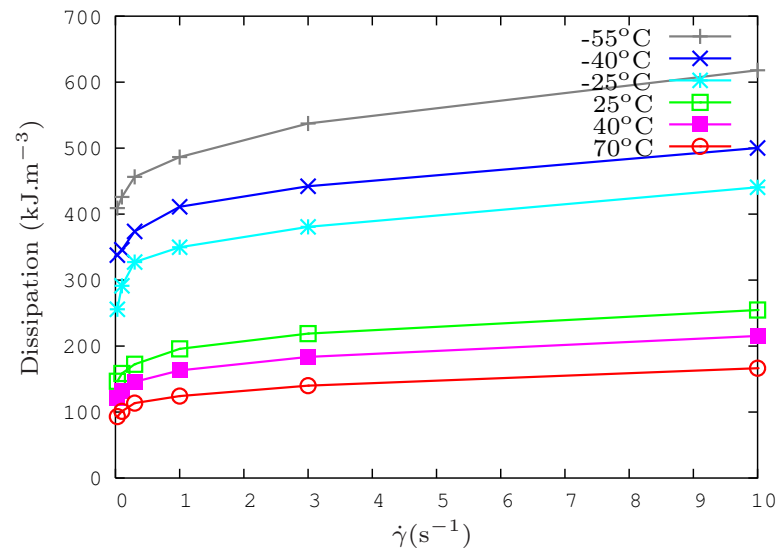

(b) Cyclic dissipation vs strain rate

Figure 6: Effects of the strain rate on the response of the material under triangular cyclic shearing tests at various temperatures $\left(\gamma_{\max }=50 \%\right)$.

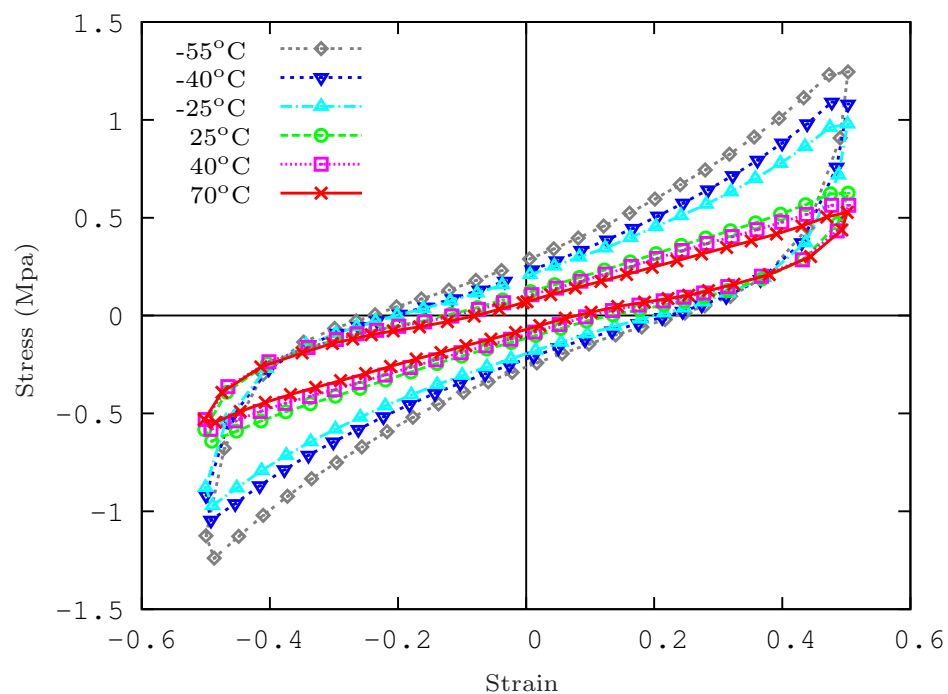

Figure 7: Effects of the temperature on the response of the the material under triangular cyclic shearing $\left(\gamma_{\max }=50 \%\right.$, $\left.\dot{\gamma}= \pm 10 s^{-1}\right)$.

- The dissipative behavior induced by viscous effects which should be coupled to the hyperelasticity.

- The model must be able to describe the behavior in a wide range of strain rates, and in particular, to reflect the Feltcher-Gent effect.

- The effects of plasticity on the behavior, including the Payne effect in particular.

Other phenomena, such as the Mullins effect and the self-heating of the material, were also observed during in this experiments. However, these aspects will not be integrated directly into the model developed in 


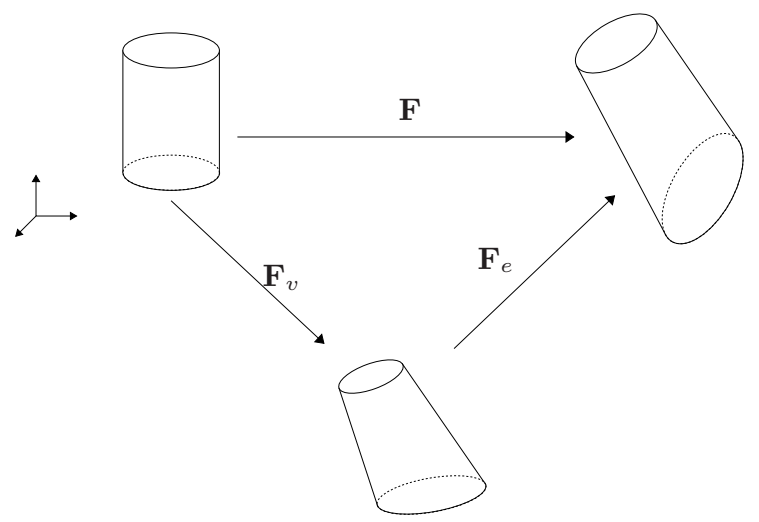

Figure 8: Intermediate configuration.

study, because their analysis has been widely discussed in the literature, see Mullins (1947, 1956, 1959); Mullins and Tobin (1965); Govindjee and Simo (1991, 1992).

\section{Constitutive equations}

\subsection{Some generalities about rheological modeling}

Using the concept of the local intermediate configuration, introduced by Sidoroff (1974, 1975), the transformation gradient tensor $\mathbf{F}$ is split into a viscous and an elastic parts:

$$
\mathbf{F}=\mathbf{F}_{e} \cdot \mathbf{F}_{v}
$$

Then, assuming that the Clausius-Duhem inequality can be written in Eulerian terms as follows (neglecting the thermal effects):

$$
\phi=\sigma: \mathbf{D}-J^{-1} \rho_{0} \dot{\psi}
$$

where $\rho_{0}$ is the density in the initial configuration, $\phi$ is the intrinsic dissipation, $\boldsymbol{\sigma}$ is the Cauchy stress tensor and $\mathbf{D}$ represents the Eulerian strain rate tensor:

$$
\mathbf{D}=\frac{1}{2}\left(\mathbf{L}+\mathbf{L}^{\mathrm{T}}\right) \quad \text { with } \quad \mathbf{L}=\dot{\mathbf{F}} \cdot \mathbf{F}^{-1}
$$

$J$ denotes the determinant of the gradient tensor $\mathbf{F}$, and $\psi$ is the free specific energy which is expressed as the sum:

$$
\psi\left(\mathbf{B}, \mathbf{B}_{e}\right)=\psi_{v}\left(\mathbf{B}_{e}\right)+\psi_{0}(\mathbf{B})
$$

where $\mathbf{B}=\mathbf{F} \cdot \mathbf{F}^{\mathrm{T}}$ and $\mathbf{B}_{e}=\mathbf{F}_{e} \cdot \mathbf{F}_{e}^{\mathrm{T}}$ are sets of independent thermodynamic variables.

So, one can express $\dot{\psi}$ as follows:

$$
\dot{\psi}=\frac{\partial \psi_{0}}{\partial \mathbf{B}}: \dot{\mathbf{B}}+\frac{\partial \psi_{v}}{\partial \mathbf{B}_{e}}: \dot{\mathbf{B}}_{e}
$$


The time derivatives of the left Cauchy-Green tensor and the local changes in volume are given by:

$$
\begin{aligned}
\dot{\mathbf{B}} & =\mathbf{L} \cdot \mathbf{B}+\mathbf{B} \cdot \mathbf{L}^{\mathrm{T}} \\
\dot{J} & =J(\mathbf{1}: \mathbf{L})
\end{aligned}
$$

where $\mathbf{1}$ is the identity tensor, and:

$$
\dot{\mathbf{B}}_{\mathbf{e}}=\mathbf{L} \cdot \mathbf{B}_{e}+\mathbf{B}_{e} \cdot \mathbf{L}^{\mathrm{T}}-2 \mathbf{V}_{e} \cdot \mathbf{D}_{v}^{o} \cdot \mathbf{V}_{e}
$$

where $\mathbf{V}_{e}$ is the purely elastic strain tensor (i.e. coming from the polar decomposition $\mathbf{F}_{e}=\mathbf{V}_{e} \cdot \mathbf{R}_{e}$ ) and

$$
\mathbf{D}_{v}^{o}=\mathbf{R}_{e} \cdot \mathbf{D}_{v} \cdot \mathbf{R}_{e}^{\mathrm{T}}
$$

is the objective measure of the anelastic strain rate. By injecting equations (6), (7) and (8) in (5), the variation of the free energy can be written as follows:

$$
\dot{\psi}=\left(2 \mathbf{B} \cdot \frac{\partial \psi_{0}}{\partial \mathbf{B}}\right): \mathbf{D}+\left(2 \mathbf{B}_{e} \cdot \frac{\partial \psi_{v}}{\partial \mathbf{B}_{e}}\right): \mathbf{D}-\left(2 \mathbf{V}_{e} \cdot \frac{\partial \psi_{v}}{\partial \mathbf{B}_{e}} \cdot \mathbf{V}_{e}\right): \mathbf{D}_{v}^{o}
$$

with the incompressibility conditions:

$$
\mathbf{D}: \mathbf{1}=0, \quad \mathbf{D}_{v}^{o}: \mathbf{1}=0
$$

Using the assumption of the normal dissipation, (we choose a quadratic pseudo-potential of dissipation, $\varphi_{v}$, depending only on $\mathbf{D}_{v}^{o}$ ), the constitutive equation and the evolution law are obtained as follows ${ }^{2}$ :

$$
\begin{aligned}
& \boldsymbol{\sigma}=\boldsymbol{\sigma}_{\mathbf{0}}+\boldsymbol{\sigma}_{\boldsymbol{v}}-p \mathbf{1} \text { with }\left\{\begin{array}{l}
\boldsymbol{\sigma}_{\mathbf{0}}=\left[2 \rho_{0} J^{-1} \mathbf{B} \cdot \frac{\partial \psi_{0}}{\partial \mathbf{B}}\right]^{\mathrm{D}} \\
\boldsymbol{\sigma}_{\boldsymbol{v}}=\left[2 \rho_{0} J^{-1} \mathbf{B}_{e} \cdot \frac{\partial \psi_{v}}{\partial \mathbf{B}_{e}}\right]^{\mathrm{D}}
\end{array}\right. \\
& \frac{\partial \varphi_{v}}{\partial \mathbf{D}_{v}^{o}}=\left[2 \rho_{0} J^{-1} \mathbf{V}_{e} \cdot \frac{\partial \psi_{v}}{\partial \mathbf{B}_{e}} \cdot \mathbf{V}_{e}\right]^{\mathrm{D}}
\end{aligned}
$$

where $p$ is the hydrostatic pressure due to the local incompressibility condition. Equations (12) and (13) can be said to be a generalization of the classical Zener rheological model to the case of finite strain.

\subsection{A statistical approach for a hyper-visco-plastic model}

The constitutive model must first reflect the behavior of the material in a wide range of strain rates, but it also has to account for the effects of the plasticity, such as the Payne effect, and the behavior of the material at low temperatures. Previous studies have shown that plasticity gives good agreement between the

\footnotetext{
${ }^{2}$ the symbol ${ }^{\mathrm{D}}$ stands for the deviatoric operator.
} 


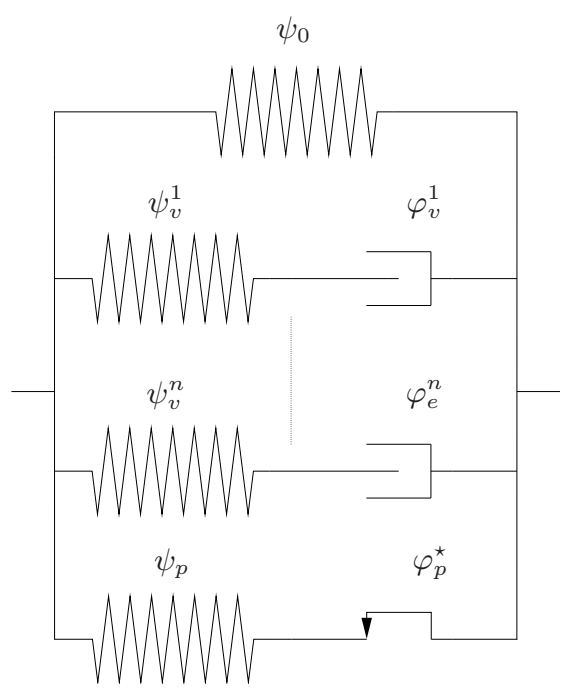

(a) Discrete statistical model.

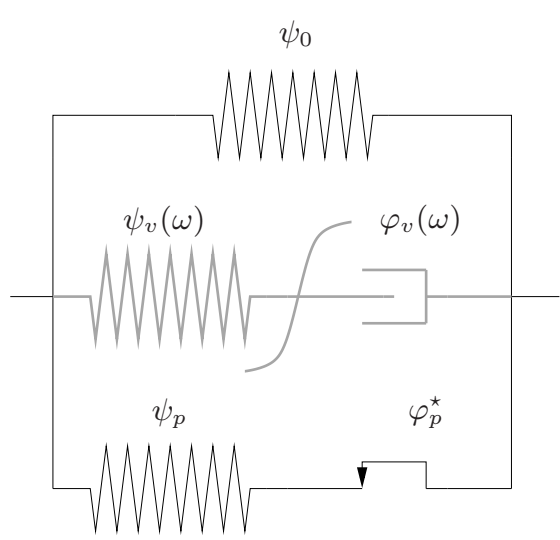

(b) Continuous statistical model.

Figure 9: Statistical hyper-visco-plastic model

experimental data and the model at low temperatures (Boukamel et al. (2005)), and that some rheological models are suitable for modeling the behavior under a given range of loads (Olsson and Austrell (2001); Miehe and Keck (2002); Nedjar (2002)). Under more complex loading conditions, rheological models with several branches (see Figure 9(a)) seem to account satisfactorily for the behavior of the material. However, the disadvantage of these models is that they require identifying a large number of parameters.

In order to overcome this difficulty, a statistical approach was developed, whereby the assembly of discrete rheological branches is extended to a continuous model with an infinite number of branches. The advantage of this method is that it covers a wide range of retardation times (or a large frequency spectrum). This approach gives the advantages of a multi-branch assembly without increasing the number of parameters in the model.

The model presented here, can be motivated in micro-physical terms by the heterogeneity of reinforced elastomers, especially in the case of a silicone elastomer slightly filled with silica particles. In order to account for this heterogeneity, it is therefore assumed that the elastomeric matrix is dense and that the inclusions, which consist of particles of silica agglomerated together with a thin rubber bond, are supposed to be slightly reticulated (see for instance Drozdov and Dorfmann (2003)). Based on these assumptions, the behavior of the elastomer can be defined as follows:

- The behavior of elastomeric matrix is hyperelastic ;

- The behavior of the inclusions is hyper-visco-elastic (an extension of the Maxwell model to large strains); 
- The inclusion/matrix interfaces is assumed to have a hyper-elasto-plastic behavior (an extension of the Saint-Venant model to large strains).

Two statistic quantities are introduced, namely:

- $\omega_{i}$, which denotes the activation energy of the mechanism inclusion/matrix, i.e. the energy required to break the links at the interface (Drozdov (2000)),

- and $\mathcal{P}_{i}$ which represents the probability of that a population of inclusion corresponds to the activation energy $\omega_{i}$.

The discrete form of the statistical model can be written:

$$
\left\{\begin{array}{l}
\psi=\psi_{0}(\mathbf{B})+\sum_{i=1}^{N} \tilde{\psi}_{v}\left(\omega_{i}, \mathbf{B}_{e}\left(\omega_{i}\right)\right) \mathcal{P}_{i}+\psi_{p}\left(\mathbf{B}_{\mathbf{e p}}\right) \\
\varphi=\sum_{i=1}^{N} \tilde{\varphi}_{v}\left(\omega_{i}, \mathbf{D}_{v}^{o}\left(\omega_{i}\right)\right) \mathcal{P}_{i}+\varphi_{p}^{\star}\left(\boldsymbol{\sigma}_{\mathbf{p}}\right)
\end{array}\right.
$$

where $\mathbf{D}_{p}^{o}$ denotes the anelastic objective strain rate of the elasto-plastic branch (same definition as in equation (9)), $\psi_{0}$ denotes the specific free energy associated with the matrix, whereas $\tilde{\psi}_{v}$ and $\psi_{p}$ are the free energies associated with the inclusions and the inclusions/matrix interfaces, respectively. $\tilde{\varphi}_{v}$ and $\varphi_{p}^{\star}$ are the pseudo-potential of dissipation ${ }^{3}$ corresponding to the inclusions and to the interface, respectively.

Using the continuous statistical model in Figure 9(b) to generalize this formulation, we obtain:

$$
\left\{\begin{array}{l}
\psi=\psi_{0}(\mathbf{B})+\int_{0}^{\infty} \tilde{\psi}_{v}\left(\omega, \mathbf{B}_{e}(\omega)\right) P(\omega) d \omega+\psi_{p}\left(\mathbf{B}_{\mathbf{e p}}\right) \\
\varphi=\int_{0}^{\infty} \tilde{\varphi}_{v}\left(\omega, \mathbf{D}_{v}^{o}(\omega)\right) P(\omega) d \omega+\varphi_{p}^{\star}\left(\boldsymbol{\sigma}_{\mathbf{p}}\right)
\end{array}\right.
$$

where $\omega$ is a random variable associated with the activation energy of a relaxation micromechanism, and $P(\omega)$ is the probability that a population of fillers has the given value $\omega$.

Substituting the potentials (15) in equations (12) give the constitutive equation of the statistical model ${ }^{4}$ :

$$
\boldsymbol{\sigma}=\boldsymbol{\sigma}_{\mathbf{0}}+\int_{0}^{\infty} \tilde{\boldsymbol{\sigma}}_{\boldsymbol{v}}(\omega) P(\omega) d \omega+\boldsymbol{\sigma}_{\mathbf{p}}-p \mathbf{1} \text { with }\left\{\begin{array}{l}
\boldsymbol{\sigma}_{\mathbf{0}}=2 \rho_{0} J^{-1}\left[\mathbf{B} \cdot \frac{\partial \psi_{0}}{\partial \mathbf{B}}\right]^{\mathrm{D}} \\
\tilde{\boldsymbol{\sigma}}_{\boldsymbol{v}}(\omega)=2 \rho_{0} J^{-1}\left[\mathbf{B}_{e}(\omega) \cdot \frac{\partial \tilde{\psi}_{v}(\omega)}{\partial \mathbf{B}_{e}(\omega)}\right]^{\mathrm{D}} \\
\boldsymbol{\sigma}_{\mathbf{p}}=2 \rho_{0} J^{-1}\left[\mathbf{B}_{\mathbf{e p}} \cdot \frac{\partial \psi_{p}}{\partial \mathbf{B}_{\mathbf{e p}}}\right]^{\mathrm{D}}
\end{array}\right.
$$

\footnotetext{
${ }^{3}$ The symbol * denotes a Legendre-Fenchel transformation.

${ }^{4}$ In the hyperelasto-plastic branch, $\mathbf{F}$ is split into an elastic part $\mathbf{F}_{\mathbf{e p}}$ and a plastic part $\mathbf{F}_{\mathbf{p p}},\left(\mathbf{F}=\mathbf{F}_{\mathbf{e p}} \cdot \mathbf{F}_{\mathbf{p p}}\right)$. The objective measure of the plastic strain rate $\mathbf{D}_{p}^{o}$ is therefore defined in the same way as $\mathbf{D}_{v}^{o}$ (see (9)).
} 
and the following evolution laws:

$$
\begin{aligned}
\frac{\partial \tilde{\varphi}_{v}(\omega)}{\partial \mathbf{D}_{v}^{o}(\omega)} & =2 \rho_{0} J^{-1}\left[\mathbf{B}_{e}(\omega) \cdot \frac{\partial \tilde{\psi}_{v}(\omega)}{\partial \mathbf{B}_{e}(\omega)}\right]^{\mathrm{D}} \\
\mathbf{D}_{p}^{o} & =\left[\frac{\partial \varphi_{p}^{\star}}{\partial \sigma_{\mathbf{p}}}\right]^{\mathrm{D}}
\end{aligned}
$$

The various forms of the potentials can be chosen so that: the neo-Hookean incompressible hyperelastic model for the matrix behavior; the neo-Hookean form, for the hyperelasticity of the inclusions and the inclusions/matrix interface; a quadratic form for the pseudo-potential of viscous dissipation of the inclusions; and a perfectly plastic pseudo-potential at the interface. These choice can be written as follows:

$$
\left\{\begin{array}{l}
\rho_{0} \psi_{0}=C_{1}\left(I_{1}(\mathbf{B})-3\right) \\
\rho_{0} \tilde{\psi}_{v}(\omega)=G(\omega)\left(I_{1}\left(\mathbf{B}_{e}(\omega)\right)-3\right) \\
\tilde{\varphi}_{v}(\omega)=\frac{\eta(\omega)}{2} \mathbf{D}_{v}^{o}: \mathbf{D}_{v}^{o} \\
\rho_{0} \psi_{p}=A_{p}\left(I_{1}\left(\mathbf{B}_{\mathbf{e p}}\right)-3\right) \\
\varphi_{p}^{\star}=<\left\|\boldsymbol{\sigma}_{\mathbf{p}}\right\|-\chi>
\end{array}\right.
$$

where $C_{1}$ is the coefficient of the neo-Hookean density, $G(\omega)$ and $\eta(\omega)$ are two functions of the random variable $\omega, \chi$ and $A_{p}$ are the parameters involved in the hyper-elasto-plastic branch, $\langle$.$\rangle denotes the$ Mac-Cauley brackets and $\left\|\sigma_{\mathrm{p}}\right\|=\sqrt{\sigma_{\mathrm{p}}: \sigma_{\mathrm{p}}}$.

To define the distribution function $P(\omega)$, a classical Gaussian distribution centred at the origin and characterized by the standard deviation $\Omega$ was adopted:

$$
P(\omega)=\frac{1}{\int_{0}^{\infty} P(\omega) d \omega} \operatorname{Exp}\left[-\left(\frac{\omega}{\Omega}\right)^{2}\right]
$$

To choose the functions $G(\omega)$ and $\eta(\omega)$, which describe the variations in the hyperelastic and viscous characteristics depending on $\omega$, several forms were tested. The functions giving the best match with the experimental data were of the form:

$$
\left\{\begin{array}{l}
G(\omega)=G_{0} \operatorname{Exp}[\omega], \\
\eta(\omega)=\eta_{\infty}\left[\frac{\ln [\sqrt{\omega}+1]}{\omega}+1\right]
\end{array}\right.
$$

In fact, these expressions for the hyperelastic and viscous characteristics lead to a decreasing evolution of the retardation time depending on $\omega$. Combining this variation with the distribution function (20) makes it possible to focus on the instantaneous elastic response rather than on the delayed response. 


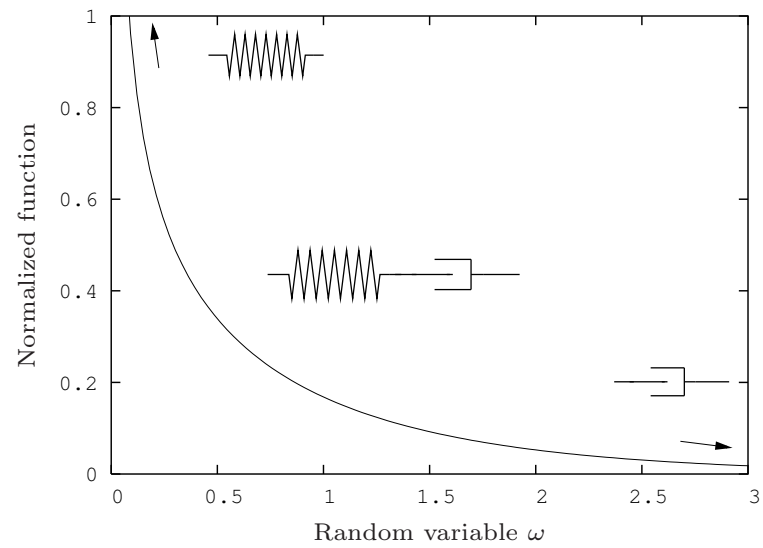

(a) Evolution of $\tau(\omega)=\frac{\eta(\omega)}{2 G(\omega)}$.

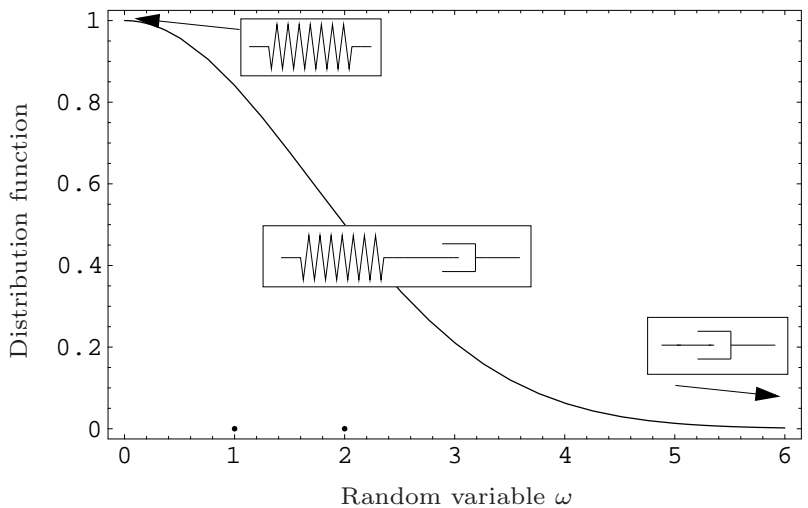

(b) Evolution of the distribution function $\mathcal{P}(\omega)$.

Figure 10: Evolution of statistical functions: predominance of the instantaneous elasticity.

Lastly, by injecting (19) in (16), we obtain the constitutive equation:

$$
\boldsymbol{\sigma}=\boldsymbol{\sigma}_{\mathbf{0}}+\int_{0}^{\infty} \tilde{\boldsymbol{\sigma}}_{\boldsymbol{v}}(\omega) P(\omega) d \omega+\boldsymbol{\sigma}_{\mathbf{p}}-p \mathbf{1} \text { with }\left\{\begin{array}{l}
\boldsymbol{\sigma}_{\mathbf{0}}=2 C_{1} \mathbf{B}^{\mathrm{D}} \\
\tilde{\boldsymbol{\sigma}}_{\boldsymbol{v}}(\omega)=2 G(\omega) \mathbf{B}_{e}(\omega)^{\mathrm{D}} \\
\\
\boldsymbol{\sigma}_{\mathbf{p}}=2 A_{p} \mathbf{B}_{\mathbf{e p}}^{\mathrm{D}}
\end{array}\right.
$$

and by using (19), (17) and (18) in (8), we obtain the following flow rules:

$$
\begin{aligned}
\dot{\mathbf{B}}_{\mathbf{e}}(\omega) & =\mathbf{L} \cdot \mathbf{B}_{e}(\omega)+\mathbf{B}_{e}(\omega) \cdot \mathbf{L}^{\mathrm{T}}-4 \frac{G(\omega)}{\eta(\omega)} \mathbf{B}_{e}(\omega) \cdot \mathbf{B}_{e}(\omega)^{\mathrm{D}} \\
\dot{\mathbf{B}}_{\mathbf{e p}} & =\mathbf{L} \cdot \mathbf{B}_{\mathbf{e p}}+\mathbf{B}_{\mathbf{e p}} \cdot \mathbf{L}^{\mathrm{T}}-2<\left\|\boldsymbol{\sigma}_{\mathbf{p}}\right\|-\chi>\frac{\boldsymbol{\sigma}_{\mathbf{p}}}{\left\|\boldsymbol{\sigma}_{\mathbf{p}}\right\|} \cdot \mathbf{B}_{\mathbf{e p}}
\end{aligned}
$$

The statistical hyper-visco-plastic model given by expressions (22) therefore includes 6 parameters which have to be identified, 5 of which are determinist parameters $\left(C_{1}, G_{0}, \eta_{\infty}, A_{p}, \chi\right)$ and one of which is a statistical parameter $(\Omega)$.

\section{Identification of the model parameters}

To identify the model parameters by fitting the response of the model to the experimental data, an algorithm implemented in the MATHEMATICA software was used. This algorithm, is based on a minimization of the sum of the squared differences between the experimental data and the analytical or semi-analytical responses. The latter are obtained by simulating the uniaxial tension tests and the double shear tests, in which the responses are assumed to be homogeneous and incompressible. 


\subsection{Analytical forms of tension responses}

Under uniaxial tension, the elastic and anelastic gradients of the transformation are written as follows, respectively:

$$
\mathbf{F}=\left[\begin{array}{ccc}
\lambda & 0 & 0 \\
0 & \frac{1}{\sqrt{\lambda}} & 0 \\
0 & 0 & \frac{1}{\sqrt{\lambda}}
\end{array}\right], \quad \mathbf{F}_{a}=\left[\begin{array}{ccc}
\lambda_{a} & 0 & 0 \\
0 & \frac{1}{\sqrt{\lambda_{a}}} & 0 \\
0 & 0 & \frac{1}{\sqrt{\lambda_{a}}}
\end{array}\right]
$$

Substituting these expressions into the constitutive equations and the complementary law (22), the response of the material under various loading modes can be obtained using an analytical form or after a numerical solving.

\subsubsection{Relaxation test}

To obtain the relaxation response, the instantaneous and delayed stresses are written in terms of the axial component of the first Piola-Kirchoff stress tensor $\Pi,\left(\sigma=\Pi_{11}\right)$ :

$$
\left\{\begin{array}{l}
\sigma_{0}=2\left(\lambda^{3}-1\right)\left[\left(C_{1}+\int_{0}^{\infty} G(\omega) P(\omega) d \omega\right) \frac{1}{\lambda^{2}}\right]+\sqrt{\frac{3}{2}} \frac{\chi}{\lambda} \\
\sigma_{\infty}=2 \frac{C_{1}}{\lambda^{2}}\left(\lambda^{3}-1\right)+\sqrt{\frac{3}{2}} \frac{\chi}{\lambda} \\
\left.\dot{\sigma}\right|_{t=0}=-\frac{8}{3}\left(2 \lambda^{3}-\lambda-\frac{1}{\lambda^{3}}\right) \int_{0}^{\infty} \frac{G(\omega)^{2}}{\eta(\omega)} P(\omega) d \omega
\end{array}\right.
$$

where $\sigma_{0}$ is the instantaneous stress response and $\sigma_{\infty}$ is the infinite (long-time) stress response.

\subsubsection{Hardening test}

The axial stress is written here in the quasi-static case, in the form:

$$
\sigma(\lambda)=\frac{2}{\lambda}\left[\frac{C_{1}}{\lambda}\left(\lambda^{3}-1\right)+A_{p} \frac{\lambda_{e}^{3}-1}{\lambda_{e}}\right]
$$

The plastic and elastic elongations $\lambda_{p}$ and $\lambda_{e}$ are given by:

$$
\dot{\lambda}_{p}=<\dot{\lambda}>\mathcal{H}\left(\lambda-\lambda_{y}\right) \quad \text { and } \quad \lambda_{e}=\frac{\lambda}{\lambda_{p}}
$$

where $\mathcal{H}$ is a hardening function which is obtained from eq. (24) $\lambda_{y}$ is the elongation corresponding to the plastic yield:

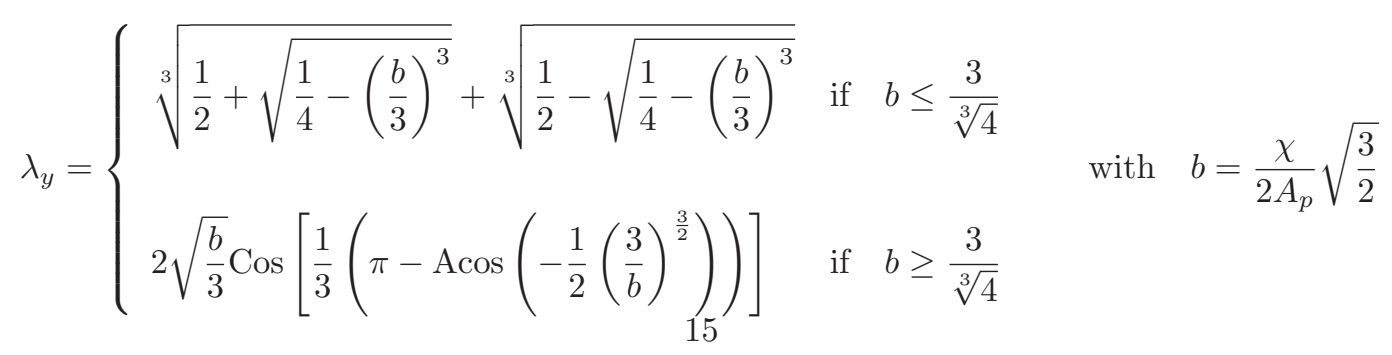


Lastly, the residual strain at zero stress $\lambda_{0}$ corresponds to the equation $\sigma\left(\lambda_{0}\right)=0$ when $\dot{\lambda}<0$, which can be written as a function of $\left(C_{1}, A_{p}, \chi\right)$ and the maximum strain.

\subsection{Shear responses}

In the case of shear tests, the gradient tensors are taken to be as follows:

$$
\mathbf{F}=\left[\begin{array}{ccc}
1 & \gamma & 0 \\
0 & 1 & 0 \\
0 & 0 & 1
\end{array}\right], \quad \mathbf{F}_{a}=\left[\begin{array}{ccc}
\lambda_{a_{1}} & \gamma_{a} & 0 \\
0 & \lambda_{a_{2}} & 0 \\
0 & 0 & \frac{1}{\lambda_{a_{1}} \lambda_{a_{2}}}
\end{array}\right]
$$

\subsubsection{Relaxation test}

With the approximation, $\lambda_{a_{i}}=1$, the instantaneous and delayed stress relaxation terms $\left(\tau=\Pi_{12}\right)$ are given by:

$$
\left\{\begin{array}{l}
\tau_{0}=2 \gamma\left(C_{1}+\int_{0}^{\infty} G(\omega) P(\omega) d \omega\right)+\frac{\sqrt{2}}{2} \chi \\
\tau_{\infty}=2 C_{1} \gamma+\frac{\sqrt{2}}{2} \chi \\
\dot{\tau}_{t=0}=-8 \gamma \int_{0}^{\infty} \frac{G(\omega)^{2}}{\eta(\omega)} P(\omega) d \omega
\end{array}\right.
$$

\subsubsection{Hardening test}

The response of the material under quasi-static loading/unloading can be approximated as follows. The shearing stress is written:

$$
\tau(\gamma)=2 C_{1} \gamma+2 A_{p} \gamma_{e}
$$

The plastic and elastic shear strain $\gamma_{p}$ and $\gamma_{e}$ are given by:

$$
\dot{\gamma}_{p}=<\dot{\gamma}>\mathcal{H}\left(\gamma-\gamma_{y}\right) \quad \text { and } \quad \gamma_{e}=\gamma-\gamma_{p}
$$

where $\gamma_{y}$ is the shear strain corresponding to the plastic yield:

$$
\gamma_{y}=\sqrt{\frac{\sqrt{9+\frac{3 \chi^{2}}{2 A_{p}^{2}}}-3}{2}} \simeq \frac{\sqrt{2} \chi}{4 A_{p}}
$$

\subsubsection{Cyclic test}

More generally, based on expressions (30), the response to a cyclic shear test can be obtained by writing the constitutive equations (22). This leads to a system of differential equations, the solutions of which are $\left\{\gamma_{a}, \lambda_{a_{1}}, \lambda_{a_{2}}\right\}$. These systems can be solved using a Runge-Kutta scheme. 


\subsection{Identification algorithm}

The identification of the parameters of the model $\mathbf{X}=\left\{C_{1}, G_{0}, \eta_{\infty}, A_{p}, \chi, \Omega\right\}$ can be reduced to the minimization of the difference between the experimental curves $\left\{\left(\lambda_{i}, \sigma_{i}\right), i=1, N_{T}\right\}$ and $\left\{\left(\gamma_{i}, \tau_{i}\right), i=1, N_{S}\right\}$ and the theoretical responses $(\lambda, \sigma(\lambda, \mathbf{X}))$ and $(\gamma, \tau(\gamma, \mathbf{X}))$. This difference is characterized by the least square distance:

$$
E(\mathbf{X})=\sqrt{\sum_{i=1}^{N_{T}} \xi_{i}\left(\sigma_{i}-\sigma\left(\lambda_{i}, \mathbf{X}\right)\right)^{2}+\sum_{i=1}^{N_{S}} \eta_{i}\left(\tau_{i}-\tau\left(\gamma_{i}, \mathbf{X}\right)\right)^{2}},
$$

where $\xi_{i}$ and $\eta_{i}$ are the weights associated with the tensile and shear tests, respectively.

This minimization problem is solved using Powell's iterative algorithm (see Fletcher (1987)), which is a conjugate direction method without gradient calculation. This algorithm is combined with a one-dimensional minimization procedure in each direction which is based on a quadratic interpolation of the function to be minimized.

In short, the identification procedure consists of:

1. determining of the response of the material with a set of model parameters, under a given loading mode;

2. calculating the least square difference between the modeling predictions and the experimental data;

3. applying the iterative procedure to minimize the least square difference.

\subsection{Identification strategy}

Given the complexity of the model, the number of parameters which have to be identified and the multiplicity of the experimental data required to identify these parameters, it was necessary to develop a strategy for decoupling the various stages in the identification procedure. This identification strategy was based on the distinction between the instantaneous and delayed responses, as well as between the effects of viscosity and plasticity. Based on the analytical or semi-analytical results outlined in the previous paragraphs, the following strategy was therefore adopted:

1. Quasi-static and delayed responses are used to identify the parameters $C_{1}, A_{p}$ and $\chi$.

2. The other parameters, $G_{0}, \Omega$ and $\eta_{\infty}$, can be obtained by fitting the values to the instantaneous or cyclic responses at various strain rates.

Table (1) summarizes the successive steps in the identification strategy.

\section{Results and Discussion}

\subsection{Identification results}

The six model parameters $\left(C_{1}, G_{0}, \eta_{\infty}, A_{p}, \chi, \Omega\right)$ were identified successively, at various temperatures $\left(-55^{\circ} \mathrm{C},-40^{\circ} \mathrm{C},-25^{\circ} \mathrm{C}, 25^{\circ} \mathrm{C}, 40^{\circ} \mathrm{C}\right.$ and $\left.70^{\circ} \mathrm{C}\right)$ and at various strain rates $\left(3 s^{-1}, 10 s^{-1}\right)$. The values obtained are given in table (2). 


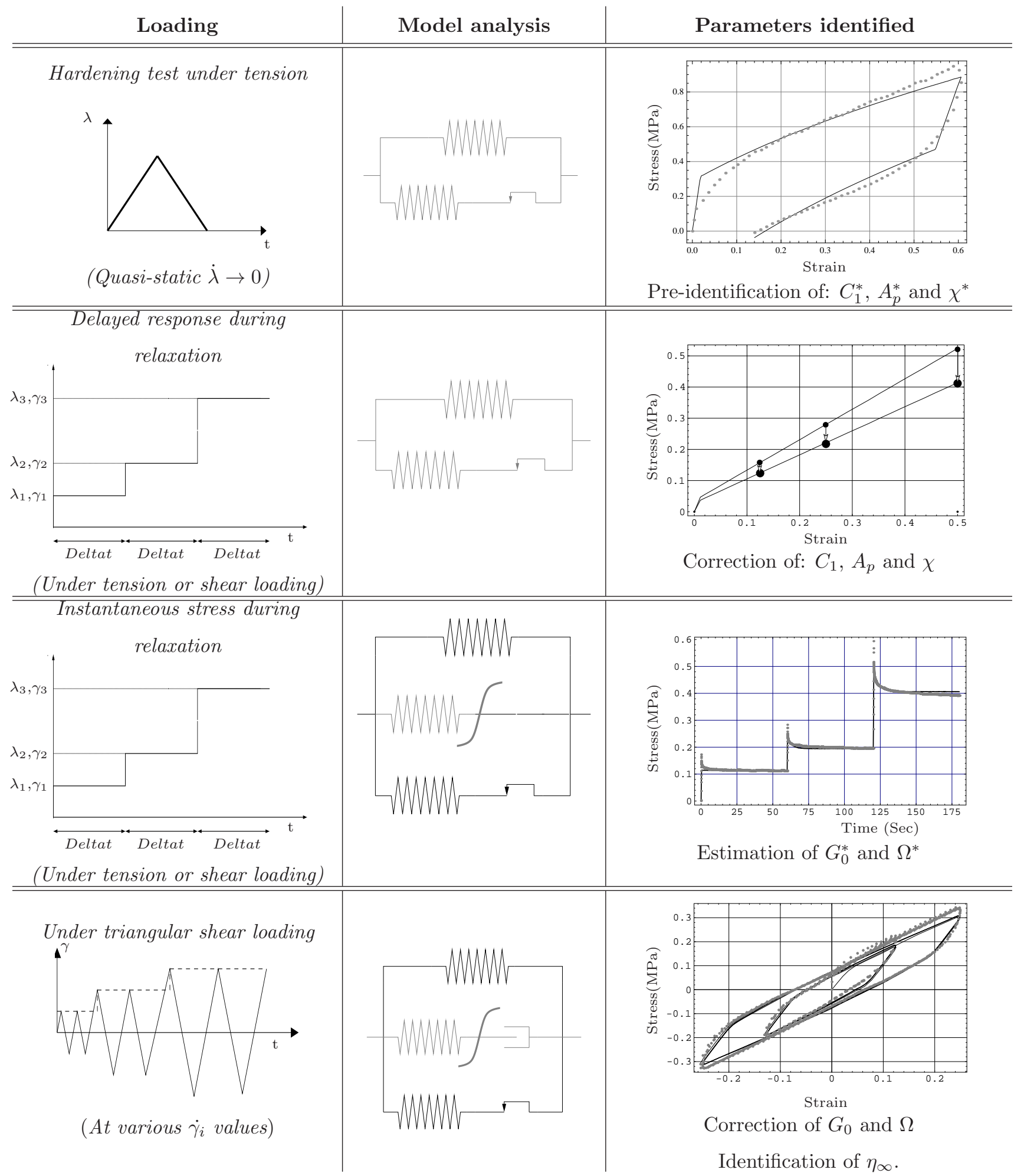

Table 1: Identification strategy: $C_{1}, A_{p}$ and $\chi$ are identified on quasi-static and delayed responses, $G_{0}, \Omega$ and $\eta_{\infty}$ are obtained by fitting the values to the instantaneous or cyclic responses at various strain rates. 


\begin{tabular}{r||c|c|c|c|c|c||c|c}
\multicolumn{1}{c|}{$\mathrm{T}\left({ }^{\circ} \mathrm{C}\right)$} & $c_{1}(\mathrm{MPa})$ & $a_{p}(\mathrm{MPa})$ & $\chi(\mathrm{Mpa})$ & $a_{0}(\mathrm{MPa})$ & $\eta_{\infty}(\mathrm{MPa} . \mathrm{s})$ & $\Omega$ & $3 s^{-1}$ & $10 s^{-1}$ \\
\hline \hline-55 & 0.538 & 2.424 & 0.107 & 0.151 & 0.14 & 1.56 & 10.84 & 12.43 \\
\hline-40 & 0.486 & 2.109 & 0.088 & 0.136 & 0.105 & 1.56 & 12.45 & 12.17 \\
\hline-25 & 0.451 & 1.767 & 0.075 & 0.121 & 0.096 & 1.56 & 15.28 & 11.82 \\
\hline 25 & 0.386 & 1.23 & 0.04 & 0.0614 & 0.063 & 1.56 & 7.98 & 8.75 \\
\hline 40 & 0.359 & 1.024 & 0.031 & 0.059 & 0.056 & 1.56 & 7.78 & 6.79 \\
\hline 70 & 0.342 & 0.855 & 0.02 & 0.051 & 0.053 & 1.56 & 10.15 & 6.86
\end{tabular}

Table 2: Parameters identified at various temperatures.

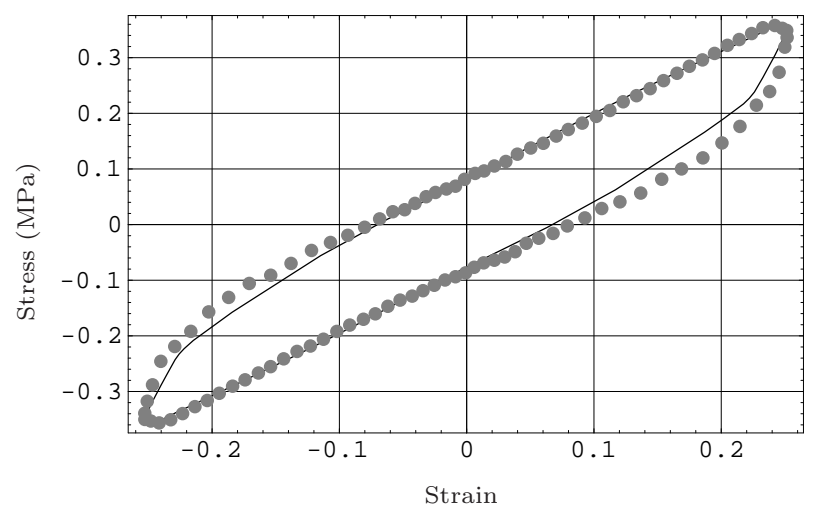

(a) Strain rates: $10 s^{-1}, \mathrm{~T}=25^{\circ} \mathrm{C}$

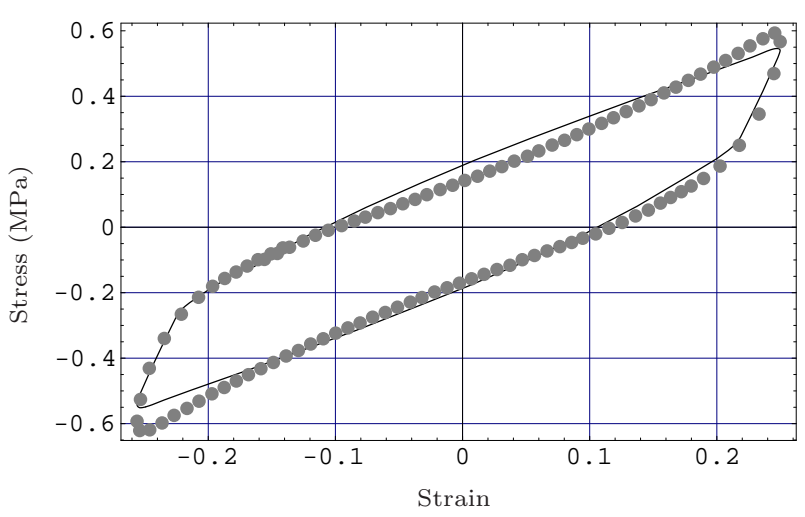

(b) Strain rate: $3 s^{-1}, \mathrm{~T}=-55^{\circ} \mathrm{C}$

Figure 11: Comparison between stabilized hysteretic cycles at various temperatures and strain rates. The solid line show the model response, the points show the experimental results.

The table (2) also gives the relative identification error obtained for each temperature and each strain rate. A maximum of $15 \%$ of error is obtained and the figure 11 shows two examples of identification results. A good agreement between the predictions of the model and the experimental data is observed in the shear tests, at various temperatures and strain rates.

Lastly, Figure 12 shows the evolution of the model parameters identified (normalized) versus the temperature. These curves suggest an exponential decay of all the parameters with the increase of the temperature, except for $\Omega$, which has been fixed at all temperatures, since it characterized only the range of retardation times to be covered by the model. This result shows that the present model seems consistent as the evolution of the parameters between the temperature is monotonous in the temperature range considered.

\subsection{Relevance of the model}

Comparisons between the results of the model predictions and the experimental data (which has not been used for identification), obtained under sinusoidal shear loading conditions, show that the model accurately 


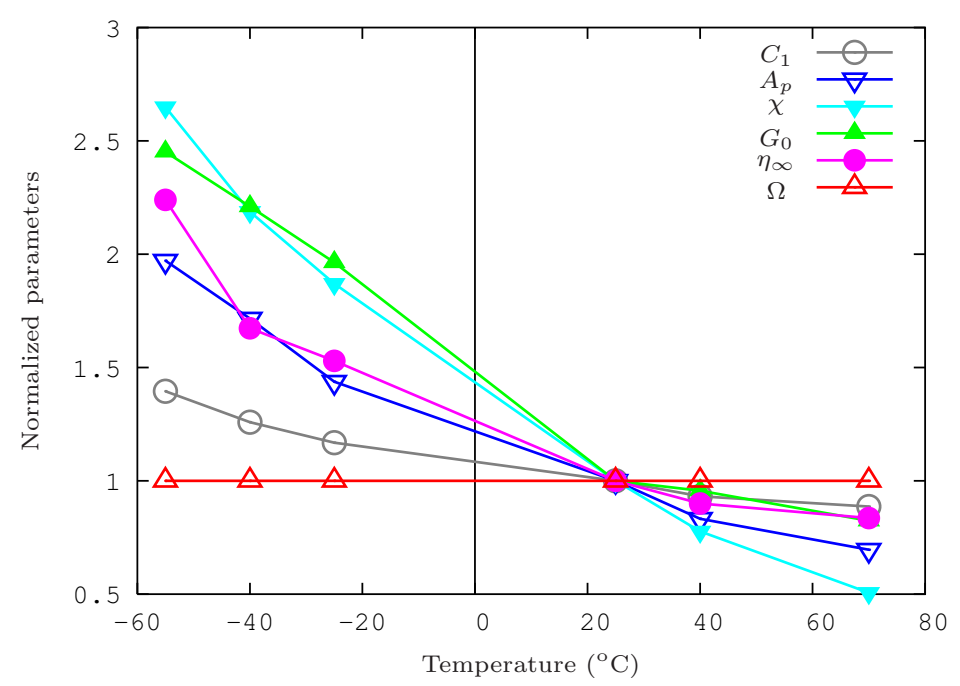

Figure 12: Evolution of parameters vs temperature.

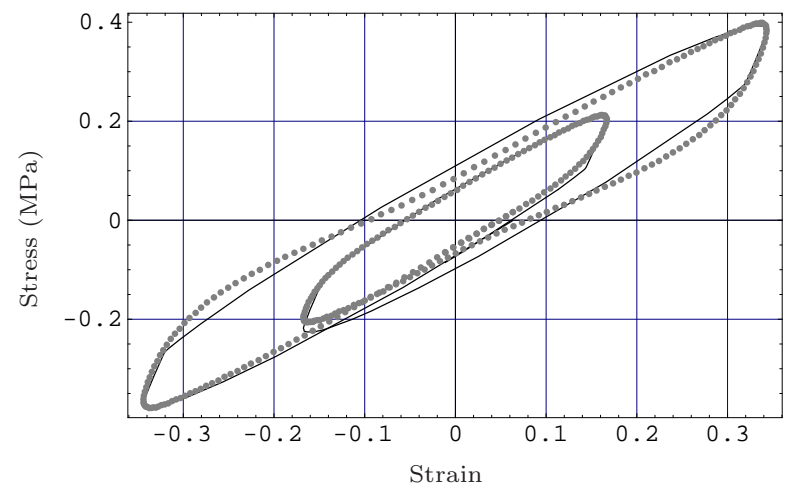

(a) $\gamma(t)=0.125 \sin (2 \pi \times 1.5 \times t)+0.25 \sin (2 \pi \times 3 \times t)$

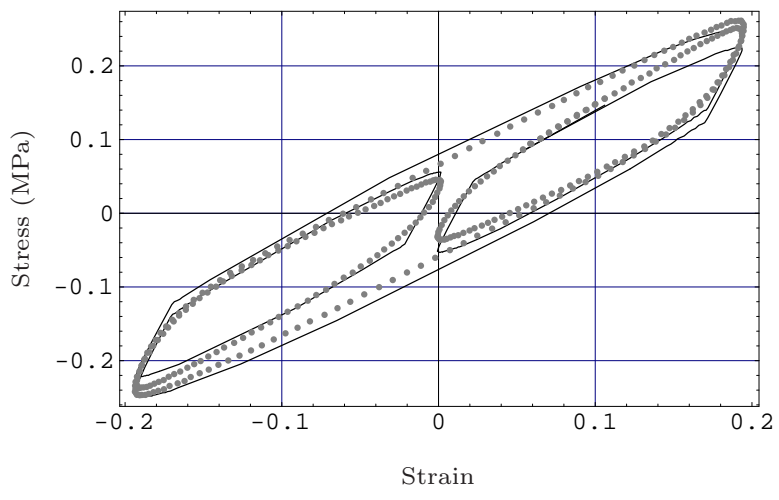

(b) $\gamma(t)=0.125 \sin (2 \pi \times 1.5 \times t)+0.125 \sin (2 \pi \times 4.5 \times t)$

Figure 13: Comparison of the global response of the model (solid line) and experimental data (points) in bi-harmonic shear test.

predicts the effects observed experimentally, namely, the Payne effect (see Harwood et al. (1967)), as shown in Figure 14(c), and The Gent-Fletcher effect (see Fletcher and Gent (1953)) as shown in Figure 14(a). In addition, figures $14(\mathrm{~b})$ and $14(\mathrm{~d})$ show the existence of good agreement between the simulated and the experimental data on the cyclic dissipation depending on the frequency and the amplitude.

Other comparisons made in multi-harmonic loading in shear tests also shows the ability of this model to accurately simulate the behavior of materials subjected to a combination of several loads at different frequencies (see figure (13)). These results show that the present model can successfully predict the complex behavior of a highly dissipative silicone rubber for a large range of strain amplitudes and strain rates with 


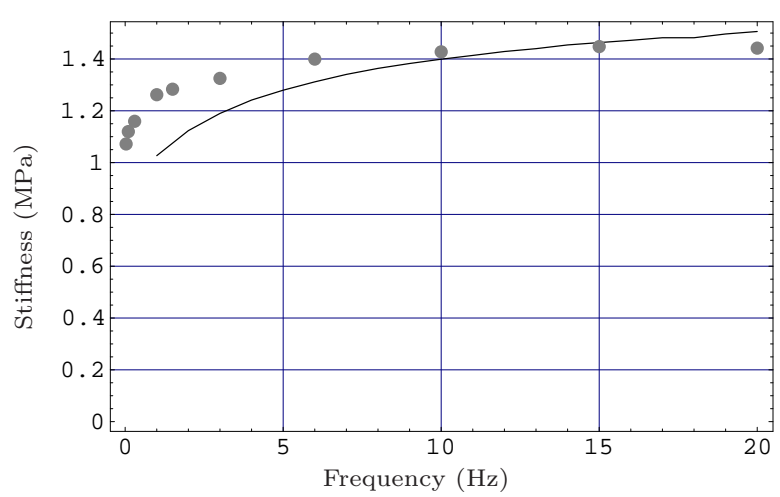

(a) Global stiffness vs frequency

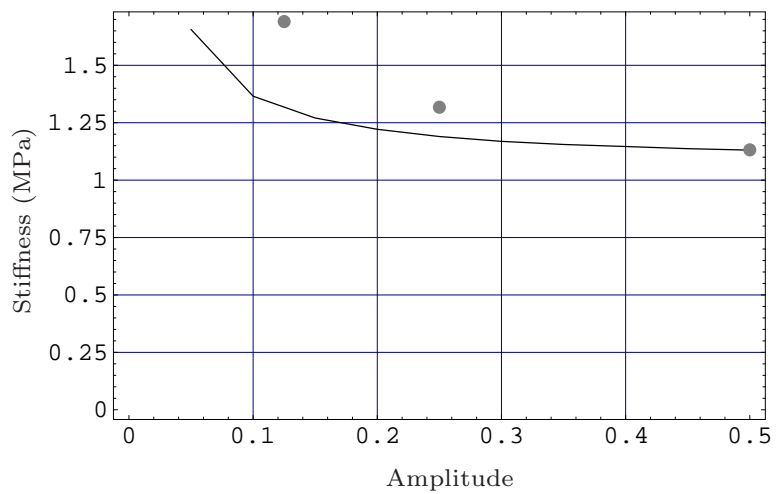

(c) Global stiffness vs amplitude

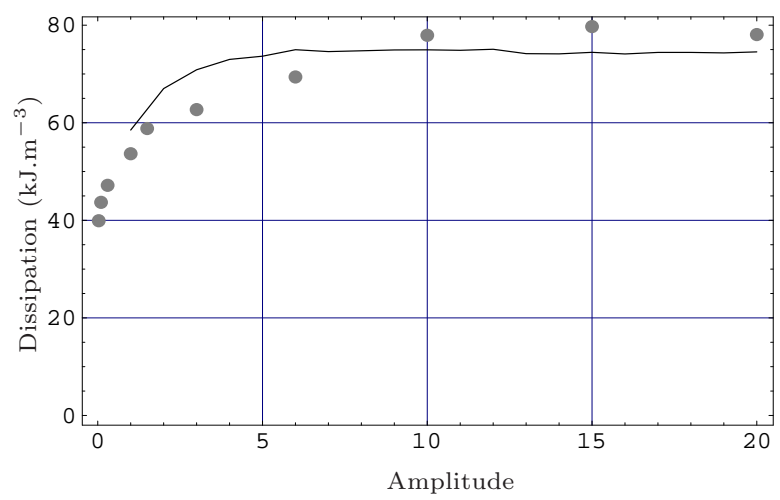

(b) Dissipation vs frequency

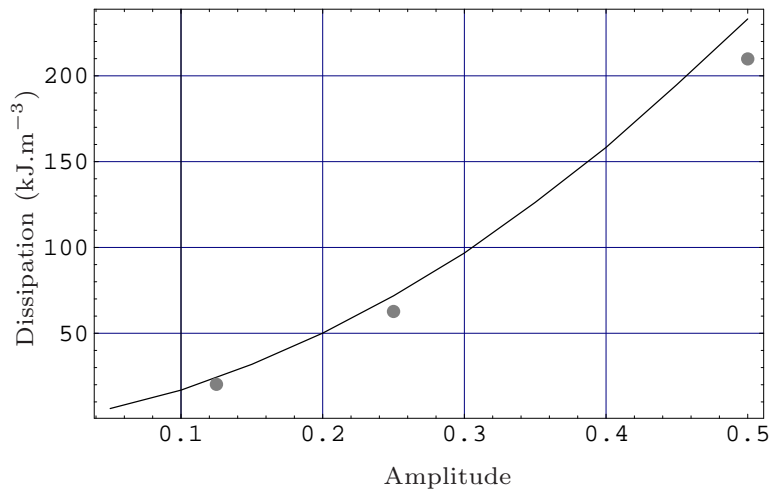

(d) Dissipation vs amplitude

Figure 14: Comparisons between modeling predictions (solid line) and experimental data (points).

a few number of material parameters.

\section{Conclusion}

In this study, a statistical approach was used to develop a hyper-visco-plastic model covering a wide frequency range. This approach has the same advantages of classical multi-branch models, such as the ability to simulate the behavior of material for several decades of retardation time, but it do not show the same inconvenient as only a few number of material parameters are required (6 in the present model).

A series of experiments were conducted under various loading and temperature conditions, in order to identify the parameters involved in the model, using an algorithm developed with the MATHEMATICA software library. To optimize this identification procedure, a relevant strategy was adopted, which consisted in distinguishing between the various stages in the procedure and thus reducing the effects of coupling 
between the parameters.

The results obtained at various temperatures show the ability of this model to simulate the behavior of the material in a wide range of temperatures. In addition, the comparisons between the modeling predictions and the experimental data recorded at various frequencies and strain amplitudes have shown a good agreement. The present model is therefore capable to reproduce the complex behavior of filled rubber in particular the Gent-Fletcher and Payne effects.

\section{References}

Boukamel, A., Martinez, J., Méo, S., 2005. A statistical approach for a hyper-visco-elasto-plastic model for a filled elastomer. In: Constitutive Models for Rubber IV. Proceedings of the 4th European Conference for Constitutive Models for Rubber, pp. 191-196.

Chang, W., Bloch, R., Tschoegl, N., 1978. The behaviour of rubber-like materials in moderatly large deformations. Journal of Rheology 22, 1-32.

Christensen, R., 1971. Theory of viscoelasticity, an introduction. Academic Press Inc., New York.

Coleman, B., 1964. Thermodynamics of materials with memory. Arch. Rational Mech. Anal 17, 1-46.

Coleman, B., Noll, W., 1961. Foundations of linear viscoelasticity. Rev. Mod. Phys. 33, 239-249.

Drozdov, A., 1998. A model for the nonlinear viscoelastic response in polymers at finite strains. International Journal of Solids and Structures vol. 35 (18), p. 2315-2347.

Drozdov, A., 2000. A model of cooperative relaxation in finite viscoelasticity of amorphous polymers. Int. J. Non-Linear Mechanics 35, 897-909.

Drozdov, A., 2001a. A model for the viscoelastic and viscoplastic responses of glassy polymers. International Journal of Solids and Structures 38, 8285-8304.

Drozdov, A., 2001b. Modelling nonlinear viscoelasticity and damage in amorphous glassy polymers. Mathematical and Computer Modelling 33, 883-893.

Drozdov, A., Agarwal, S., Gupta, R., 2004. Linear thermo-viscoelasticity of isotactic polypropylene. Computational Materials Science 29, 195-213.

Drozdov, A., Dorfmann, L., 2002. The nonlinear viscoelastic response of carbon black-filled naturals rubbers. International Journal of Solids and Structures vol. 39, p. 5699-5717.

Drozdov, A. D., Dorfmann, L., 2003. A micro-mechanical model for the response of filled elastomers at finite strains. International Journal of Plasticity vol. 19, p. 1037-1067.

Fletcher, R., 1987. Pratical methods of optimization. John Wiley \& Sons.

Fletcher, W., Gent, A., 1953. Non-linearity in the dynamic properties of vulcanised rubber compounds. I.R.I Transctions, $266-280$.

Govindjee, S., Simo, J., 1991. A micro-mechanically based continumm damage model for carbon black-filled rubbers incorporating mullins'effect. J. Mech. Phys. Solids 39, 87-112.

Govindjee, S., Simo, J., 1992. Trasnsition from micro-mechanics to computationally efficient phenomenology: carbon black filled rubbers incorporating mullins' effect. J. Mech. Phys. Solids 40, 213-233.

Hart-Smith, L., 1966. Elasticity parameters for finite deformations of rubber-like materials. J. Appl. Math. Phys. 17, 608-626. Harwood, J., Mullins, L., Payne, A., 1967. Stress softening in rubbers : a review. Journal of the IRI, 17-27.

Holzapfel, G., Reiter, G., 1995. Fully coupled thermomechanical behaviour of viscoelastic solids treated with finite elements. Int. J. Engng. Sci. 33, 1037-1058. 
Holzapfel, G., Simo, J., 1996a. Entropy elasticity of isotropic rubber-like solids at finite strains. Comput. Methods Apll. Mech. Engrg. 132, 17-44.

Holzapfel, G., Simo, J., 1996b. A new viscoelastic constitute model for continuous media at finite thermomechanical changes. Int. J. Structures 33, 3019-3034.

Le Tallec, P., 1990. Numerical analysis of viscoelastic problems. Masson.

Le Tallec, P., Rahier, C., 1994. Numerical models of steady rolling for non-linear viscoelastic structures in finite deformations. Int. J. Numer. Methods Eng. 37, 1159-1186.

Lemaître, J., Chaboche, J., 1996. Mécanique des matériaux solides. Dunod, Paris.

Leonov, A., 1992. On thermodynamics and stability of general maxwell-like viscoelastic constitutive equations, theoretical and applied rheology. In: XIth Int. Cong. On Rheology. pp. 97-99.

Lianis, G., 1963. Constitutive equations of viscoelastic solids under finite deformation. Purdue University report A\&ES 63-11.

Lion, A., 1997. On the large deformation behaviour of reinforced rubber at different temperatures. J. Mech. Phys. Solids 45, 1805-1834.

Miehe, C., 1995. Discontinuous and continuous damage evolution in ogden-type large-strain elastic materials. Eur. J. Mech., A/Solids $14,697-720$.

Miehe, C., Keck, J., 2002. Surimposed finite elastic-viscoelastic-plastoelastic stress response with damage in filled rubbery polymers. experiments, modelling and algorithmic implementation. Journal of Mechanics and Physics Solids 48, 323-365.

Mooney, M., 1940. A theory of large elastic deformation. J. Appl. Phys. 11, 582-592.

Morman, K., 1988. Original contributions. an adaptation of finite linear viscoelasticity theory for rubber-like by use of the generalised strain measure. Rheologica Acta 27, 3-14.

Mullins, L., 1947. Effect of strectching on the properties of rubber. Journal of Rubber Research 16, $275-289$.

Mullins, L., 1956. Determination of degree of crosslinking in natural rubber vulcanizates. part i. J. Poly. Sci. 19, 225-236.

Mullins, L., 1959. Determination of degree of crosslinking in natural rubber vulcanizates. part iii. J. Appl. Poly. Sci. 2, 1-7.

Mullins, L., Tobin, N., 1965. Stress softening in rubber vulcanizates. part i, use of a strain amplification factor to describe the elastic of filler-reinforced rubber. J. Appl. Poly. Sci. 9, 2933-3009.

Nedjar, B., 2002. Frameworks for finite strain viscoelastic-plasticity based on multiplicative decomposition. part i: Continuum formulations. Comput. Methods Appl. Mech. Engrg 191, 1541-1562.

Ogden, R., 1972. Large deformation isotropic elasticity, on the correlation of theory and experiment for incompressible rubberlike solids. Proc. Roy. Soc., Lond. A326, 565-584.

Olsson, A., Austrell, P., 2001. A fitting procedure for a viscoelastic-elastoplastic material. In: Constitutive Models for Rubber II. Proceedings of the 2nd European Conference for Constitutive Models for Rubber.

Reese, S., 2003. A micromechanically motivated material model for the thermo-viscoelastic material behaviour of rubber-like polymers. International Journal of Plasticity 19, 909-940.

Rivlin, R., 1958. Some topics in finite elasticity. In: 1st Symp. on Naval Struct. Mech.

Sidoroff, F., 1973. The geometrical concept of intermediate configuration and elastic finite strain. Arch. Mech. 25 (2), $299-309$.

Sidoroff, F., 1974. Un modèle viscoélastique non linéaire avec configuration intermédiaire. J. Méc. 13 (4), $679-713$.

Sidoroff, F., 1975. Variables internes en viscoélasticité, 2. milieux avec configuration intermédiaire. J. Méc. 14 (4), $571-595$.

Sidoroff, F., 1977. Rhéologie non-linéaire et variables internes tensorielles. Symposium franco-polonais, Cracovie.

Simo, J., 1987. On a fully three-dimensional finite-strain viscoelastic damage model : fomulation and computational aspects. Comput. Methods Apll. Mech. Engrg. 60, 153-163.

Tanaka, F., Edwards, S., 1992. Viscoelastic properties of physically cross-linked networks. transient network theory. Macromolecules 25, 1516-1523.

Treloar, L., 1943. The elasticity of a network of long chain molecules i. Trans. Faraday Soc. 39, 36-64. 
Treloar, L., 1957. The present status of the theory of large elastic deformations. In: The rheology of elastomers. 